\title{
Microwave assisted synthesis of tripodal triazines from 1,3,5-tris(2- hydroxyethyl)-1,3,5-triazinane-2,4,6-trione and fatty acids
}

José Crecente-Campo, Silvia Fernández-Sánchez, Julio A. Seijas,* M. Pilar VázquezTato*

Departamento de Química Orgánica. Facultad de Ciencias. Universidad de Santiago de Compostela, Campus de Lugo. Aptdo. 280. 27080-Lugo. Spain.

Abstract: A new fast and direct synthesis for tripodal fatty acid esters of tris-hydroxyethyl isocyanurate 1 under microwave irradiation is developed. The method is valid for saturated and unsaturated acids, but the last produced a mixture of esters cis and trans.

$$
\text { 1,3,5-tris(2-hydroxyethyl)-1,3,5-triazinane-2,4,6-trione (1), a compound }
$$
commonly used in the preparation of polymers, presents a $C_{3}$ symmetry (Figure 1), which makes it a good template to build fatty acid esters with a tripodal structure (Figure 2).

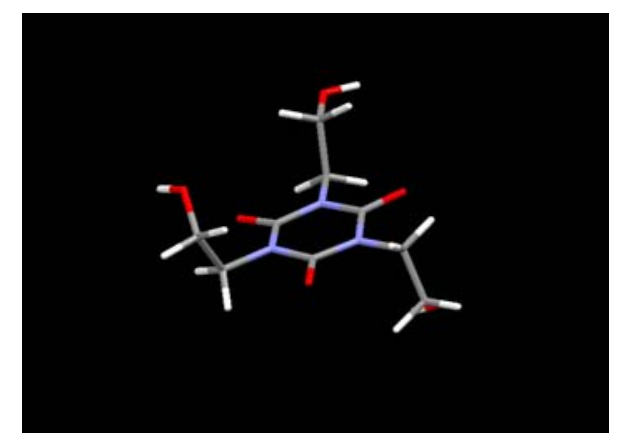

Figure 1. X-ray structure of 1,3,5-tris(2hydroxyethyl)-1,3,5-triazinane-2,4,6-trione $(1)^{1}$<smiles>[R]C(=O)OCCn1c(=O)n(CCOC([R])=O)c(=O)n(CCOC([R])=O)c1=O</smiles>

Figure 2

These esters have been reported as fibre treatment agents to improve resistance, ${ }^{2}$ lubricants, ${ }^{3}$ low viscosity waxes, ${ }^{4}$ melt flow improvers ${ }^{5}$ and heat-resistant finishes for synthetic fibers. ${ }^{6}$ All the previous reports on these compounds used conventional esterification methods, usually with long reaction times.

Microwave assisted organic synthesis (MAOS) seemed to be a good candidate to improve the preparation of these esters. Previous reports have demonstrated the usefulness of this tool in esterification reactions. ${ }^{7}$ Then, we decided to study the esterification of $\mathbf{1}$ by microwave irradiation, under solvent-free conditions with solid $p$ - 
toluenesulfonic acid as catalyst. Thus, a 1:3 mixture of $\mathbf{1}$ and decanoic acid together with a catalytic amount of $\mathrm{TsOH}(1.67 \mathrm{~mol} \%)$ were heated to $160^{\circ} \mathrm{C}$ for 10 minutes yielding 2,2',2"-(2,4,6-trioxo-1,3,5-triazinane-1,3,5-triyl)tris(ethane-2,1-diyl) tris(decanoate) (2) in 85\%. (Scheme 1).<smiles>O=c1n(CCO)c(=O)n(CCO)c(=O)n1CCO</smiles>

1<smiles>CCCCC(=O)O</smiles>

$\underset{160^{\circ} \mathrm{C}, \mathrm{MW}}{\stackrel{p-\mathrm{TsOH}, 1.67 \mathrm{~mol} \%}{\longrightarrow}}$

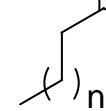

$\mathrm{n}=7, \mathrm{~T}=160^{\circ} \mathrm{C}$ $\mathrm{n}=15, \mathrm{~T}=110^{\circ} \mathrm{C}$

$2, \mathrm{n}=7,85 \%$

$3, n=15,52 \%$

Scheme 1

Under the same reaction conditions stearic acid suffered pyrolysis. Thus, temperature was decreased in steps of 10 degrees, finding that at $110^{\circ} \mathrm{C}$ there was no carbonization yielding, after 20 minutes, a 52\% of 2,2',2"-(2,4,6-trioxo-1,3,5-triazinane1,3,5-triyl)tris(ethane-2,1-diyl) tristearate (3) (Scheme 1).

In order to check the stability of double bonds oleic acid ((9Z)-Octadec-9-enoic acid) was studied. Irradiation for 30 minutes at $160^{\circ} \mathrm{C}$ yielded $68 \%$ of a mixed triester of elaidic (trans) and oleic (cis) acids as detected by ${ }^{1} \mathrm{H}$ NMR (Figure 3), in an approximated ratio of 2:1, which could correspond to (9E,9'E)-2,2'-(5-(2-((Z)-nonadec9-enoyloxy)ethyl)-2,4,6-trioxo-1,3,5-triazinane-1,3-diyl)bis(ethane-2,1-diyl) dioctadec9-enoate where $\mathbf{1}$ is esterified with two molecules of elaidic acid and one of oleic acid. 


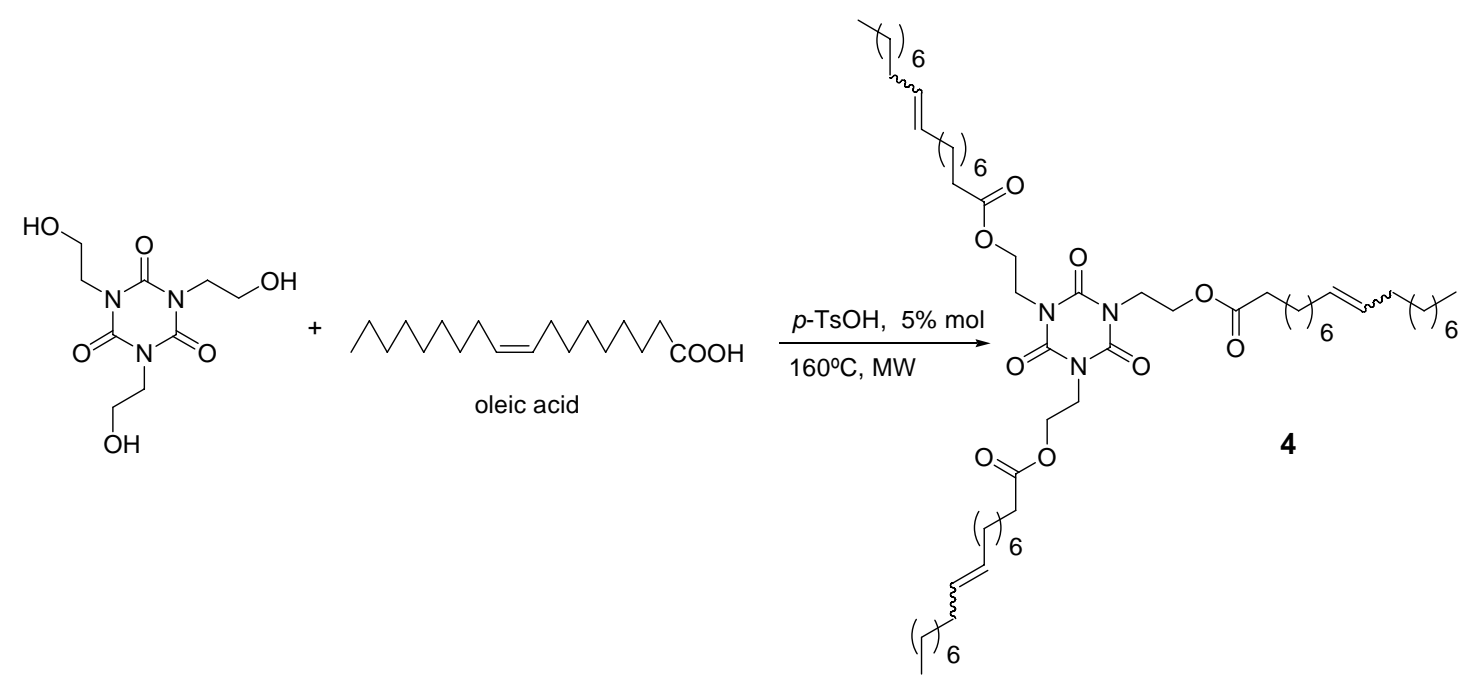

Scheme 2
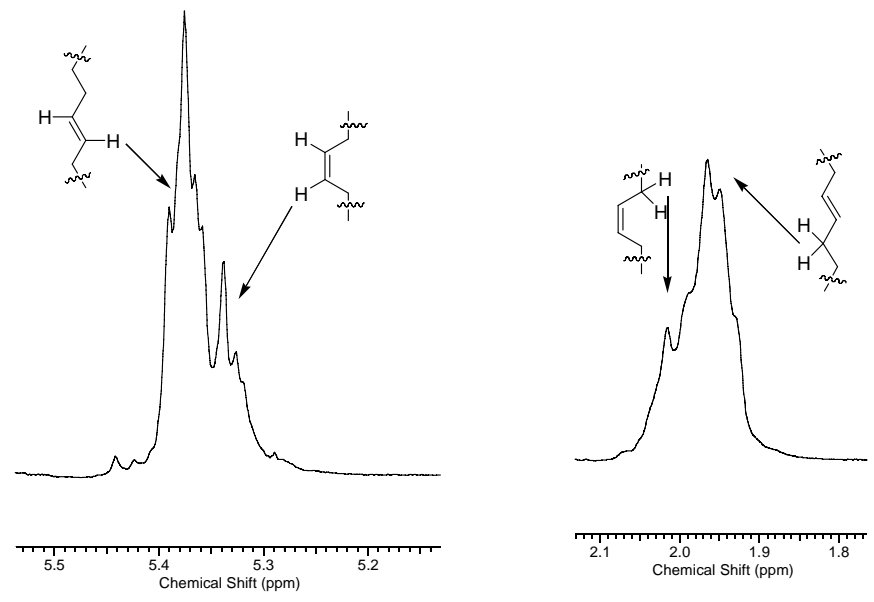

Figure 3

In summary, we developed a new fast and direct synthesis for tripodal fatty acid esters of trishydroxyethyl isocyanurate $\mathbf{1}$. This allows easy access to this kind of compounds in order to study their properties as lipid analogues (Figure 4). 


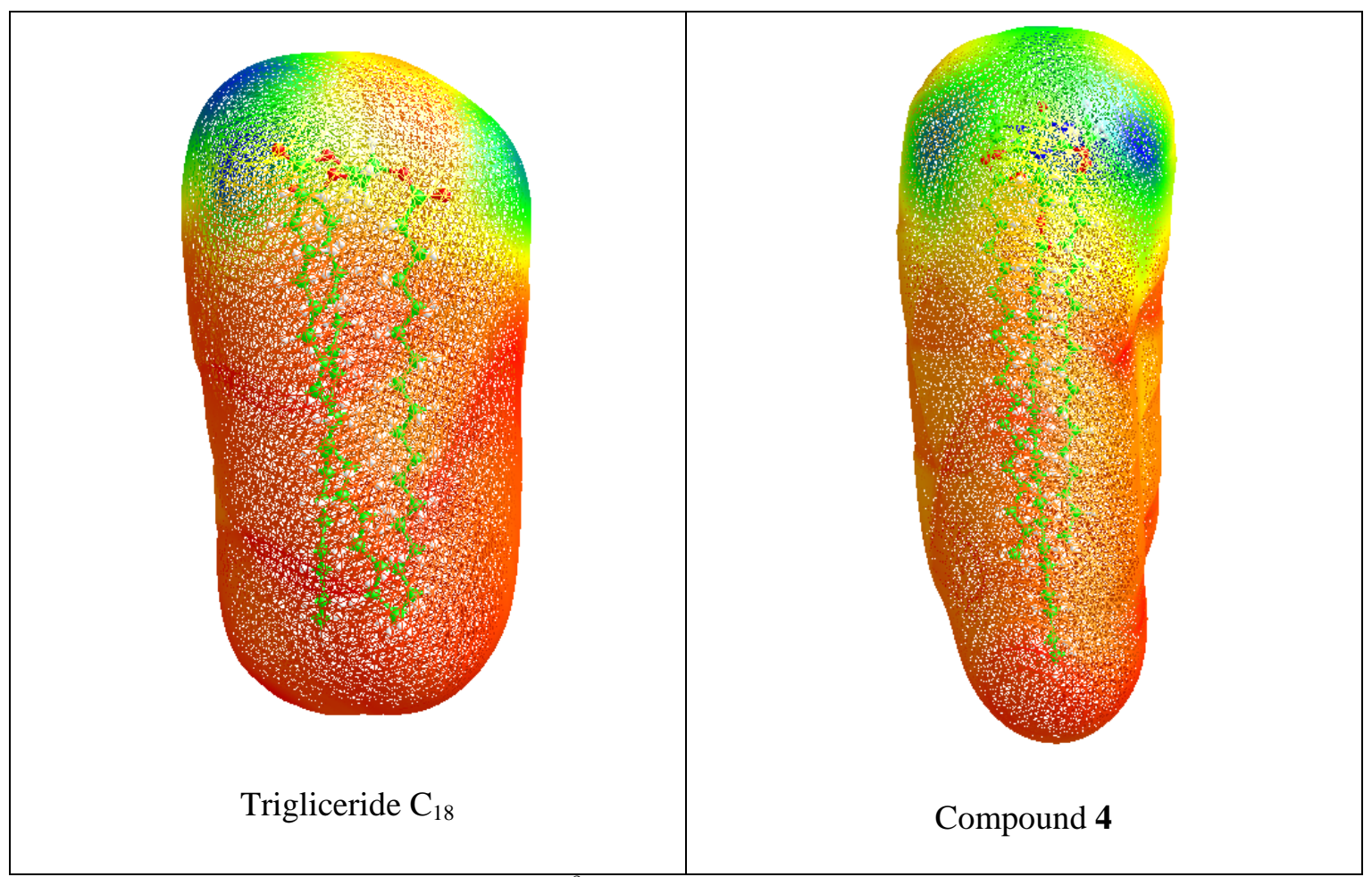

Figure 4. Molecular lipophilic potential ${ }^{8}$

\section{Acknowledgements}

XUNTA DE GALICIA for financial support: PGIDIT05PXIB26201PR and USC for a predoctoral fellowship to JCC.

\section{General experimental procedure}

Compound 1 (0.199 g, $0.76 \mathrm{mmol}$ ) thoroughly mixed with decanoic acid (0.408 g, $2.28 \mathrm{mmol})$ and $p$-TsOH $(7.3 \mathrm{mg}$ ), was irradiated in a CEM Discover monomode oven for 10 minutes (200W, $160^{\circ} \mathrm{C}$ ). The crude reaction was dissolved in $\mathrm{CH}_{2} \mathrm{Cl}_{2}$ and washed with $\mathrm{NaOH}_{\mathrm{aq}}(10 \%)$ and water. After evaporation the residue was purified by chromatography on silica column, yielding $2(0.473 \mathrm{~g}, 85 \%)$ as a thick oil.

1 Chong, S. Y.; Seaton, C. C.; Kariuki, B. M.; Tremayne M. Acta Crystallogr. ,Sect. B: Struct. Sc. 2006, $62,864$.

\footnotetext{
2 Yamakita, H.; Ito, Y. Japan Patent JP2008163489, 2008, Scifinder CAN 149:202523, 2008.

${ }^{3}$ Gan, X.; Qiu, S.; Lu, X.; Zhang, Z.; Wang, G.; Hu, Z.; Zhang, D.; Xing, Y. China Patent CN 1583985, 2005, Scifinder CAN 144:54123, 2005.
}

${ }^{4}$ Kitamura, T.; Doi, K. Japan Patent 1992 JP 04246589, Scifinder CAN 118:149815 1993 
${ }^{5}$ Nakayama, Y. Japan Patent JP 04248867 1992, Scifinder CAN 118:170302 1993

${ }^{6}$ Kitamura, K.; Hosomi, H. Japan Patent JP 62125079, 1987, Scifinder CAN 107:238521, 1987.

Minagawa, M.; Nakahara, Y. Japan Patent JP 48071444, 1973, Scifinder CAN 80:60580, 1974.

7 "Microwaves in organic synthesis" $2^{\text {nd }}$ edition, A. Loupy ed. Wiley-VCH, 2006. Sivan Velmathi, Nagahata, R.; Sugiyama, J.; Takeuchi K. Macromol. Rapid Commun. 2005, 26, 1163-1167.

${ }^{8}$ Surface calculated with software VEGA ZZ. Pedretti, A.; Villa, L.; Vistoli, G. J. Comp.-Aid. Mol. Design., 2004, 18, 167-173. Pedretti, A.; Villa, L.; Vistoli, G. J. Mol. Graph., 2002, 21, 47-49. Pedretti, A.; Villa, L.; VistoliTheor G. Chem. Acc., 2003, 109, 229-32. http://www.ddl.unimi.it. The Molecular Lipophilicity Potential (MLP) is calculated projecting the Broto-Moreau lipophilicity atomic constants on the molecular surface (Gaillard, P.; Carrupt, P.A.; Testa, B.; Boudon, A. J. Comp.-Aid. Mol. Design., 1994, 8, 83). 